This is the final peer-reviewed accepted manuscript of:

Cecilia Testa, Severyn Salis, Nicola Rubattu, Paola Roncada, Roberto Miniero \& Gianfranco Brambilla (2019) Occurrence of Fipronil in residential house dust in the presence and absence of pets: a hint for a comprehensive toxicological assessment, Journal of Environmental Science and Health, Part B, 54:6, 441-448

The final published version is available online at:

http://dx.doi.org/10.1080/03601234.2019.1607133

Rights / License:

The terms and conditions for the reuse of this version of the manuscript are specified in the publishing policy. For all terms of use and more information see the publisher's website.

This item was downloaded from IRIS Università di Bologna (https://cris.unibo.it/)

When citing, please refer to the published version. 


\title{
Occurrence of Fipronil in residential house dust in presence and absence of pets: a hint for a comprehensive toxicological assessment
}

\author{
CECILIA TESTA ${ }^{1}$, SEVERYN SALIS ${ }^{1}$, NICOLA RUBATTU ${ }^{1}$, PAOLA RONCADA $^{2}$, ROBERTO \\ MINIERO $^{3 a}$, AND GIANFRANCO BRAMBILLA*3b
}

${ }^{1}$ Istituto Zooprofilattico Sperimentale della Sardegna, Department of Food Security, Sassari, Italy

${ }^{2}$ Department of Veterinary Medical Sciences, University of Bologna, via Tolara di Sopra 50, 40064 Ozzano Emilia (BO), Italy
${ }^{3}$ Istituto Superiore di Sanità, ${ }^{a}$ Environment and Health Dept.; ${ }^{b}$ Veterinary Public Health, Food Safety, and Nutrition Dept. Viale Regina Elena, 299 I-00161 Rome, Italy

\begin{abstract}
:
The presence of the insecticide Fipronil and its main products of toxicological relevance, namely, Sulfone and Desulfinyl, was assessed in 161 residential house dust samples in the absence $(\mathrm{N}=101)$ and presence $(\mathrm{N}=60)$ of cats and dogs in Italy. High-resolution mass spectrometry analysis revealed a significant difference $(\mathrm{p}<0.001)$ in the dust contamination in the presence of pets (median: $467 \mathrm{vs}$ $24 \mathrm{ng} / \mathrm{g}$ dry weight), even if the highest value was found in the absence of pets (82,069 vs 67,799 ng/g dry weight). Fipronil intake estimates from dust in toddlers, computed according to US-EPA and EU-ECHA guidelines, ranged from 333 - 556 and from 20-34 ng/kg per day for acute and chronic scenario, respectively. Dust seemed not able itself to lead to Fipronil overexposure with respect to acute and chronic toxicity health-based guidance values. Kittens were potentially overexposed to Fipronil under both acute $(26,076 \mathrm{ng} / \mathrm{kg}$ per day) and chronic (1,633 ng/kg per day) scenarios. The mild symptomatology associated with acute intoxication could possibly determine case
\end{abstract}


underreporting within pharmacosurveillance schemes. Its administration was estimated in 7.3-9.7 tons per year. Such a range suggests its prudent use under strict veterinary control to prevent pest resistance and ecotoxicological outcomes.

*Address correspondence to Gianfranco Brambilla, Istituto Superiore di Sanità, Veterinary Public Health, Food Safety, and Nutrition Dept. Viale Regina Elena, 299 I-00161 Rome, Italy, Phone 001 39649902839 E-mail: gianfranco.brambilla@iss.it

KEYWORDS: Fipronil, pets, house dust, intake, use, assessment

\section{Introduction}

Fipronil (F) (CAS no. 120068-37-3) is a broad-spectrum phenylpyrazole insecticide recently put into the spot of the public opinion for two main reasons: a) the impact it has on bees due to its use for seed and soil treatments against soil-dwelling arthropods and early-season leaf-feeding and sucking insect pests, together with neonicotinoids, ${ }^{[1,2]}$ and b) its unauthorized use as veterinary drug in poultry farms in the European Union that lead to the presence of residues in poultry products of potential concern for children. ${ }^{[3,4]}$ Fipronil blocks the GABA A-gated chloride channels in the central nervous system, thus provoking the pest death from a prolonged and uncontrolled neuronal stimulation. After its application, F degrades under sunlight into Fipronil Desulfinyl, which has been described as being 9-10 times more active when inhibiting the mammalian chloride channel than the insect chloride channel, possibly through the formation of Fipronil Sulfone. ${ }^{[5,6]}$ The urban use encompasses the control of ectoparasites as a veterinary medicine product in anti-flea/tick/mosquito lotions; sprays for topical use on pet hair; and to fight turf beetles, cockroaches, fleas, termites, thrips, black vine weevil and other insects as a biocidal product for households, hardy ornamentals, and nonedible ornamentals. ${ }^{\text {[7] }}$ Fipronil release in urban watersheds with its main active products (Desulfinyl and Sulfone) has 
been considered recently for its ecotoxicological impact on nontarget organisms, with particular attention paid to aquatic organisms due to its toxicological features and its wide and regular use. ${ }^{[8]}$ Dust samples collected in Northern California around individual homes revealed the presence of Desulfinyl and Sulfone in $75.5 \%$ and $67.3 \%$ the of the 441 samples analyzed against the $52.4 \%$ of the parent compound, with geometric means at 71, 78, and $60 \mathrm{ng} / \mathrm{g}$ dust, respectively. ${ }^{[9]}$ The analysis of run-off waters from urban civil wastewater treatment plants from this and other regions reported Fipronil concentrations between 14 and $45 \mathrm{ng} / \mathrm{L}$, which are above the US-EPA chronic aquatic benchmark for Fipronil of $11 \mathrm{ng} / \mathrm{L}^{[10,11]}$ and the Dutch Environmental Quality Standards for surface waters of $0.7 \mathrm{ng} / \mathrm{L} .{ }^{[12]}$

According to the National Health Authority authorization, in veterinary medicine, Fipronil can be sold without a prescription at the supermarket level as sprays and spot-on formulations at $0.05-0.10 \%$ (w/v) for dogs and cats. The posology ranges between $6.7-13.3 \mathrm{mg} / \mathrm{kg}$ pet per month with the claim of protecting animals and the indoor environment from pests. The veterinary use could be of relevance for the environmental release of Fipronil and the exposure of nontarget species for the following reasons: a) its regular use in a very large pet community in urban and urban/rural contexts (Figure 1); $\left.{ }^{[13,14]} \mathrm{b}\right)$ the handling of concentrated solutions by nonprofessional people, such as groomers and pet owners, and c) the indoor pollution in the presence of treated animals that could drive postapplication exposure in sensitive groups such as toddlers and kittens, via dust intake, as result of their mouthing and licking behavior and the fur petting of treated pets. ${ }^{[15,16]}$

The first evidence of Fipronil occurrence at ppm level in indoor house dust and its association to the presence of pets was given by Mahler et al. ${ }^{[17]}$ in 2009; 19 of 24 samples of indoor dust showed a total Fipronil concentration (the sum of the parent and main products) below $270 \mathrm{ng} / \mathrm{g}$; in the remaining five samples, concentrations ranged from 1,320 to 14,200 ng/g. All three of the residences with a dog on which a flea-control product containing Fipronil was used were among the five residences with elevated Fipronil concentrations. More recently, Starr et al. ${ }^{[18]}$ (2016), in a study about the Fipronil oral bioaccessibility from house dust, found Fipronil in the range 1-108,000 ng/g 
in 31 out of 37 samples, and Sulfone (21/37) (4-10,200 ng/g) and Desulfinyl (6/37) (4-1,900 ng/g ) were also found. The reported oral bioavailability of Fipronil ranges between the 50 and $85 \%$ of the total ingested dose, ${ }^{[19]}$ as matter of the presence of two fluoromethyl groups that may increase its absorption. ${ }^{[20]}$ Considering the quite relevant pet population in Italy targeted by potential $\mathrm{F}$ treatments (Figure 1), exploration of the association between indoor contamination and the presence of pets for a more comprehensive toxicological assessment seemed worthwhile.

\section{Materials and methods}

\section{Sampling design and analysis}

During the spring-autumn period of 2016, a convenient sampling design was set up in the Bologna District, among people regularly attending the Veterinary Teaching Hospital of Bologna University, Italy. Residential house dust samples $(\mathrm{N}=161)$ were drawn from vacuum cleaner bags, along with records about the presence of pets (cats and dogs), their body weight, and the availability of a private garden. From each vacuum bag (200 $\mathrm{g}$ of dust per bag on average), 10 individual samples of $1 \mathrm{~g}$ were drawn from different sites of the bag to form the composite sample. To avoid heterogeneity, the larger particles and sand deposits were removed, and the dust was sieved to collect the $0.2 \mathrm{~mm}$ portion.

Fipronil and its metabolites were supplied by Lab Service Analytica (Bologna, Italy), and ACN, Methanol (LiChrosolv, hypergrade for LC-MS), and other reagents and materials were purchased from Merck (Darmstadt, Germany). The analytical procedure adopted is one routinely used to determine Fipronil in food commodities, in agreement with the work of Guo et al..${ }^{[21]}$ Briefly, a test portion of 0.4 grams of dust was weighed into a $15 \mathrm{~mL}$ centrifuge tube. A volume of $1 \mathrm{~mL}$ water and $9 \mathrm{~mL}$ acetonitrile was added to each sample, the mixture was sonicated for 5 mins, vigorously mixed for $10 \mathrm{~min}$, and then centrifuged $\left(4000 \mathrm{rpm}, 5^{\circ} \mathrm{C}, 10 \mathrm{~min}\right)$. Then, $5 \mathrm{~mL}$ of extract was transferred to 
a new $15 \mathrm{~mL}$ centrifuge tube containing dispersive adsorbents (QuEChERS-based clean-up with 900 mg MgSO4, 150 mg C18, 150 mg PSA from Waters Italia, Milan, Italy) and centrifuged at $4000 \mathrm{rpm}$ for $10 \mathrm{~min}$ at $5^{\circ} \mathrm{C}$; finally $0.4 \mathrm{~mL}$ of extract was added with $0.6 \mathrm{~mL}$ Methanol and analyzed on UPLCHRMS. The chromatographic analysis of Fipronil and its products was performed using an Ultimate 3000 UPLC system (Thermo Fisher Scientific, San Jose, CA, USA). Separation was carried out on an ACQUITY BEH C18 Analytical Column (100 x $2.1 \mathrm{~mm}, 1.7 \mu \mathrm{m})$ from Waters Corporation protected by a VanGuard Pre-Column $(2.1$ x $5 \mathrm{~mm}$ ) (Waters Italia, Milan, Italy). The mobile phase consisted of (A) water and (B) methanol at a flow rate of $0.3 \mathrm{~mL} / \mathrm{min}$. The elution gradient was initially set to $10 \% \mathrm{~B}$ and ramped linearly to $50 \% \mathrm{~B}$ in $3 \mathrm{~min}$; then, it was increased to $100 \% \mathrm{~B}$ in 5 min and held for $5 \mathrm{~min}$; and finally, it was returned to the initial condition in $0.5 \mathrm{~min}$ and maintained for 2.5 min to allow column conditioning for the next injection. The injection volume was $5 \mu \mathrm{L}$. The UHPLC system was coupled to a Q-Exactive Orbitrap MS (Thermo Fisher Scientific, Bremen, Germany), which was operated with a heated electrospray interface (HESI) in negative electrospray ionization (ESI). The ion transfer capillary temperature, spray voltage, sheath gas flow rate, auxiliary gas flow rate and S-lens RF level were set to $325^{\circ} \mathrm{C}, 3.5 \mathrm{kV}, 60,30$ and 55 , respectively. The QExactive MS was tuned and calibrated in positive and negative mode once a week using the calibration solutions, including caffeine, MRFA, and a mixture of Ultramark 1621 fluorinated phosphazine. The mass spectra were acquired with full MS mode at a resolution of 70,000 FWHM with $2.0 \times 106$ of Automatic Gain Control (AGC) target and $100 \mathrm{~ms}$ of maximum ion injection time. The analyses were performed without lock mass. Data were processed using XcaliburTM3.1.66.102.2.1 and Trace Finder 3.2 software (Thermo Fisher Scientific, Les Ulis, France) was used for identifying the untargeted Fipronil Desulfinyl according to the exact mass as identification criteria; confirmation of identity was achieved by automated matching of the given and additional criteria from the HRAM MS/MS Spectra Library.

\section{Quality Control}


Linearity was investigated by analysis of the calibration curves over the concentration ranges of 0.01-20 ng/g using least squares linear regressing of peak area versus concentration. Limits of Detection (LODs) were estimated analyzing three blank samples spiked at 0.01, 0.05 and $0.1 \mathrm{ng} / \mathrm{g}$ levels. They are defined as the minimum concentration at which the accurate mass error was <5ppm; LODs resulted in $0.01 \mathrm{ng} / \mathrm{g}$ for both Fipronil and Sulfone. The limits of quantification (LOQs) were estimated based on the lowest concentration measurable $(0.05 \mathrm{ng} / \mathrm{g})$ with precision expressed in terms of calculated Intra-day Repeatability (RSDr $=11.35$ for Fipronil and 16.75 for Sulfone); Trueness was estimated with the recovery test at $10 \mathrm{ng} / \mathrm{g}($ recovery $=101 \%)$ and $100 \mathrm{ng} / \mathrm{g}($ recovery $=125 \%)$ levels. The results indicated the method was suitable for the quantification of the target analytes in considered matrix.

More detailed information is included in the supporting materials. Data for the dust were computed on a ng/g dry weight basis, as total Fipronil.

\section{Data handling and statistics}

Left censored data were computed adopting the medium bound $(1 / 2$ of the LOQ $=5 \mathrm{ng} / \mathrm{g})$ approach. The differences between i) the groups of dust collected in the absence and presence of pets and ii) within the presence of pets group, the groups reporting the presence or absence of private garden in the households were evaluated by the U-Mann-Whitney nonparametric test (Statgraphics XVI, Statpoint Technologies, Inc) The outliers taken in considerations in both the evaluations were identified by the MAD/0.6745, which is an estimate based on the median absolute deviation (the median of the absolute differences between each data value and the sample median) (Statgraphics XVI, Statpoint Technologies, Inc, supplied by Adalta, Arezzo, Italy).

\section{Fipronil use in veterinary medicine}


The estimated use of Fipronil as an anti-ectoparasitic agent in dogs and cats accounted for the following data: a) the inventory of the number of cats $(6,967,000)$ was derived from the 2018 report of the National Petfood Association, ${ }^{[13]}$, and for the number of dogs $(10,717,479)$, the 2018 official registry from the Italian Ministry of Health report on dogs was used, which reported more or less the equivalent of $1 \mathrm{dog}$ to every 6 inhabitants in Italy (Figure 1), and b) the low range posology of 6.7 $\mathrm{mg} / \mathrm{kg}$ bw per month for an average weight of adult cats $(4.42 \pm 1.55 \mathrm{~kg}$ bw, $\mathrm{N}=352)$ and dogs $(17.07$ $\pm 12.34 \mathrm{~kg}$ bw, $\mathrm{N}=1427$ ) was determined from records made on the admission of cats and dogs to the Bologna University Veterinary Small Animals Clinic. We estimated an 8-month-long treatment for ticks from the spring to autumn, with an application rate once a month per pet. To discriminate when the amount of Fipronil in house dust could be associated with treated pets and not to other nonagricultural uses, appropriate cut-off values were identified in the median $\left(50^{\text {th }}\right.$ percentile, P50) + the Median Absolute Deviation (MAD) and in the $75^{\text {th }}$ percentile (P75) contamination recovered from the dataset of house dust in absence of pets $(\mathrm{N}=101)$. The percentage of house dust samples collected in the presence of pets falling above such cut-off value was then applied to the overall pet population in Italy to achieve a reliable estimate of the $\mathrm{F}$ amount used on a yearly basis as a veterinary drug.

\section{Intake assessment via dust}

The intake assessment of dust was performed to account for the following consensus-based default values: a) average intake rate of $60 \mathrm{mg}$ of dust per day, given as a central tendency for children 1 to 6 years old by the U.S. Environmental Protection Agency; ${ }^{[22]}$ b) $100 \mathrm{mg}$ per day referred to observational data in the European Children and adopted by the European Chemical Agency; ${ }^{[23]}$ and c) the mean default body weight assigned to 2-old Italian toddlers of $12.2 \mathrm{~kg}$. For kittens, a default $200 \mathrm{mg}$ dust per day intake was assumed for an 8-week old kitten of a weight of $0.52 \mathrm{~kg}$, as already reported. ${ }^{[24,25]}$ The desorption coefficient was assumed equal to 1 , and the bioavailability posed at $85 \%$ according to evidence from in vivo oral exposure in laboratory animals. ${ }^{[19]}$ 


\section{Risk characterization}

The following Health-Based Guidance Values (HBGVs) were taken into account: an Acute Reference Dose (ARfD) of 3,000 from FAO ${ }^{[19]}$ and of 9,000 $\mathrm{ng} / \mathrm{kg}$ bw per day from EFSA ${ }^{[26]}$ and the same Acceptable Daily Intake (ADI) of $200 \mathrm{ng} / \mathrm{kg}$ bw per day considered both by FAO and EFSA. We considered the maximum and the $95^{\text {th }}$ percentile of dust contamination as total Fipronil for acute toxicity in the presence and absence of pets under a worst-case scenario. For chronic toxicity, the $75^{\text {th }}$ percentile occurrence in dust seemed the most reliable indicator because that value was less biased than the mean value from the presence of outliers in the dataset considered. The risk characterization was expressed as the Margin of Safety (MOS, ratio between the HBGV for acute and chronic toxicity and the related estimated exposure).

\section{Results}

\section{House dust contamination}

Of the overall 161 samples of house dust, 101 belonged to households in the absence of pets, and of the remaining 60 samples collected in homes with pets present, 38 samples were from houses with a private garden. The Whiskers box-plots (including the outliers) of Figure 2 show the data distributions of total Fipronil in dust in the presence and absence of pets, respectively, with net significant differences $(\mathrm{P}<0.01)$. The boxes are drawn extending from the lower quartile of the sample to the upper quartile. This is the interval covered by the middle $50 \%$ of the data values when sorted from smallest to largest. The whiskers are drawn from the edges of the box to the largest and smallest 
data values, where values were unusually far away from the box (points more than 1.5 times the interquartile range (box width) above or below the box are indicated by point symbols. The statistical descriptors on occurrence of each of the analytes, including the ones describing the box intervals, are detailed in Table 1. The significance of the Fipronil occurrence in dust referred to those households with pets only, in the presence $(\mathrm{N}=38)$ or absence $(\mathrm{N}=22)$ of a private garden fell between $>0.5$ and $<0.1$. Further details are provided in the supporting materials section.

\section{Estimated use of Fipronil as veterinary medicine}

The cut-off values (median + MAD and P75) from total Fipronil occurrence in the dust from homes without pets were computed in the range of 32.82 and $109.2 \mathrm{ng} / \mathrm{g}$, which when applied to the house dust dataset in the presence of pets $(\mathrm{N}=60)$, indicated $\mathrm{F}$ use as veterinary product in $62-82 \%$ of the considered dogs and cat population (Figure 1). The estimated amount of Fipronil used as a veterinary product per year, under the assumption of an 8-month treatment, at the lowest posology $(6.7 \mathrm{mg} / \mathrm{kg}$ per month), resulted in the range of 7.292-9.656 tons per year, with a contribution from dogs up to the $86 \%$. Further information is available in the Supporting Materials

\section{Fipronil intake via dust}

In Figure 3, the computed dust intake in toddlers under the acute - maximum value and $95^{\text {th }}$ percentile (max and P95) -, and chronic (median and $75^{\text {th }}$ percentile) scenarios relative to the total Fipronil house dust contamination in the presence and absence of pets is illustrated, whereas, in Figure 4, the assessment carried out in kittens is shown. The risk characterization for acute (worst-case scenario) and chronic exposure is reported in Table 2 under an aggregate exposure scenario that accounts for the contribution from animal petting, ${ }^{[16]}$ and for dietary intake of $F$ residues in food commodities, ${ }^{[4]}$ respectively, along with the related Margin of Safety. 


\section{Discussion}

\section{Uncertainties}

From the analytical procedure, uncertainties can be restricted to the determination of Fipronil Desulfinyl as a result of the nontarget analysis. Due to an inappreciable difference in its chromatographic behavior and instrumental response with respect to matrix interferences and with respect to the differences in Fipronil and Sulfone, in agreement with Guo et al., ${ }^{[21]}$ the postacquisition determination via TraceFinder software did not affect the reliability of the results. Notably, the Sulfinyl accounts for $10 \%$ of the total Fipronil, on average.

The contamination found in vacuum bags can be assumed to be an average of 1-2 months collection; therefore, uncertainty can be reasonably addressed toward higher intakes under the worst-case scenario, with acute toxicity for dust intakes typically occurring a few days after the Fipronil application.

The oral bioavailability of Fipronil has been assumed to be at $85 \%$, accounting for the evidence from experimentally treated animals fed on Fipronil-contaminated diets, ${ }^{[19]}$ An in vitro bioavailability study reports a lower bioavailability, with a maximum of $64 \%$ as a function of the organic carbon content in dust. ${ }^{[18]}$ In vivo evidence is considered more robust than from in vitro experiments, as a matter representative of the overall digestive array. Therefore, the uncertainties show negligible influence toward lower intake estimates.

Desulfinyl, the primary environmental metabolite (photoproduct) of $\mathrm{F}$, is reported to be 9-10 times more active than the parent compound at the chloride channel of the rat brain GABA receptorNotably, a provisional ADI (pADI) $0.00003 \mathrm{mg} / \mathrm{kg}$ bw for FD was proposed by the FAO in $1999^{[19]}$ against the consolidated ADI of $0.0002 \mathrm{mg} / \mathrm{kg}$ valid for Fipronil and Sulfone; ${ }^{[19,26]}$ Desulfinyl is not currently framed in the among residues to be monitored within food safety plans, even if it has been 
recommended for the estimation of long-term and short-term dietary intake from plant and animal commodities, ${ }^{[19]}$ and from dust, according to the evidences from this and previous papers. ${ }^{[9,17,18]}$ Regarding the estimated use of Fipronil as a veterinary product, the uncertainties can be considered quite high because the purchase of the drug is not traceable from veterinary prescriptions, and the administration of the drug is done by nonprofessionals. The consideration of an 8-month long treatment per year (winter season excluded) and the low posology chosen may suggest the real use could exceed this estimate in a local context.

\section{Fipronil occurrence in residential house dust}

This wide survey on 161 samples confirms the evidence from previous papers by Malher et al., [17] and Starr et al., ${ }^{[18]}$ that an approximate range of 10,000-100,000 ng/g of total Fipronil contamination exists in residential house dust as a consequence of indoor application and/or veterinary use of Fipronil on pets. Because Starr et al. ${ }^{[18]}$ reported a maximum level as sum of Fipronil, Sulfone, and Desulfinyl in house dust of $119,006 \mathrm{ng} / \mathrm{g}$, our worst findings of 82,069 and 67,799 ng/g in the absence and presence of pets, respectively (Figure 2, Table 1), were not considered as outliers for an acute exposure scenario. However, in this work, even if the highest contamination recorded in dust was observed in households in the absence of pets, the presence of pets resulted in significantly greater total Fipronil contamination in the dust $(\mathrm{p}<0.001)$. These chronic exposure estimates, computed on $50^{\text {th }}$ and $75^{\text {th }}$ percentiles in the presence/absence of treated pets, are relevant (Figures 3 and 4). Treated pets, in households without a private garden $(\mathrm{N}=22)$, could lead to a higher house dust contamination than that recorded in those households with a private garden $(\mathrm{N}=38)$, even if the number of observations hamper the achievement of a full significance $(\mathrm{P}=0.051$; Figure 5). Within a risk orientation, the absence of a garden, and more generally, the more time spent indoor by treated pets, can be considered as a risk factor for exposure via dust intake, because of the release of cutaneous cell debris and hair in a confined environment. This risk suggests the need to further investigate Fipronil presence in the dust from residential flats. 


\section{Estimated amount of Fipronil in veterinary medicine}

In the European Union, while sales for the agriculture use of pesticides can be recovered from official statistics, no information is available about the amounts sold for urban uses. In Italy, Fipronil is marketed in approximately 85 different commercial veterinary products, and access to the amount of each commercial product sold, which is recorded under The Periodic Safety Update Report of the European Union pharmacovigilance schemes, is restricted. On a national basis, our estimates indicate the Fipronil use in veterinary medicine is in the range of 7-10 times more than that use reported in agriculture before the implementation of restrictions in Italy due to the worldwide bee health concern. The Italian Environment Research Institute ISPRA in 2017 reported an average of 0.9 tons per year of Fipronil sold for agricultural use for the treatment of seeds in the timeframe 2009-2012, sufficient to trigger surface-water monitoring oriented toward risk prioritization due to the ecotoxicity of Fipronil and its products on aquatic biota, as a matter of its environmental persistence and bioaccumulative features. ${ }^{[27]}$ Within this framework, Fipronil sourcing from pet treatments could be strictly monitored when administered under veterinary prescription, thus improving the efficacy of the pharmacovigilance and pharmacosurveillance activities; this approach would affect the potential onset of resistance as in the case of Rhipicephalus sanguineus - a three-host dog tick found worldwide and able to complete its' entire lifecycle indoors, ${ }^{[28]}$ as well as affecting the toxicological effects on most vulnerable and sensitive nontarget organisms. [3]

\section{Toxicological evidences in humans and pets}

The quite large volumes of Fipronil used by nonprofessionals may be evident in the admission of children to antipoisoning centers. A surveillance study carried out in the United States from 2001 to 
$2007^{[7]}$ showed that of 103 Fipronil-related recorded admissions to first aid/ antipoisoning centers (Sentinel Event Notification System for Occupational Risks), the majority (76\%) had exposure in a private residence, and $37 \%$ of the admissions involved the use of pet-care products. The French agency ANSES in its opinion on Fipronil in eggs ${ }^{[3]}$ reported 1,104 accidental Fipronil-related admissions to the French Antipoisoning Center network in 2011-7. Children were involved in 30\% of the reports, which showed a typical seasonality (higher incidence in May-September period), with low severity toxicity in $85 \%$ of the cases. In Italy, in the last report of the National Antipoisoning

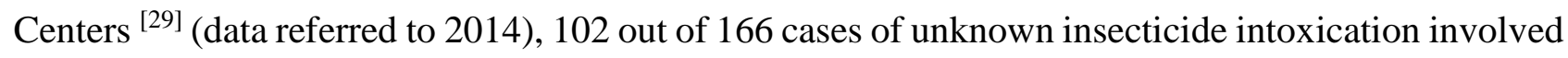
children; in this group, Fipronil was identified in 11 out of 15 cases with mild symptoms.

From our data and estimates, the impact on toddlers' health from dust intake in terms of acute and chronic toxicity does not seem sufficient to cause potential overexposure (MOS in the range of 9-16 and 6-10, respectively), without the contemporary contribution of other aggregated sources (Figure 3, Table 2). Under the acute worst-case scenario, when the $95^{\text {th }}$ percentile of the contamination values are considered, the dust intakes estimate a drop to $182-304 \mathrm{ng} / \mathrm{kg}$ bw per day in the presence of pets. However, the uncertainties related to the house dust sampling, which are not representative of recent postapplication contaminations, should be considered. For chronic exposures, the presence of Desulfinyl and its potential toxicity, along with a higher oral bioavailability of Fipronil and its products with respect to its usually exposure via the dermal route $(1 \%),{ }^{[19]}$ may deserve more attention. The computed exposures increase the uncertainties related to the Fipronil posology and appropriate application on the pets from nonprofessional people, which has led to potential higher direct and indirect exposures relevant to both acute and chronic toxicity. Its environmental release and persistence from the nonagricultural use may cause future contamination of food commodities, such as root vegetables, eggs, meat, and milk from food production animals coming in contact with Fipronil contaminated grazing areas as result of irrigation with remediated waters and/or from the use of biosolids from civil WWTPs. ${ }^{[8,30]}$ 
In the United Kingdom, veterinary pharmacovigilance reports on intoxication outbreaks in pets when Fipronil has been administered with food instead of as a pour-on. ${ }^{[31]}$ These reports stress the relevance of the oral exposure as a determinant of potential intoxication, due to its increased bioavailability via such a route. With respect to kittens as nontarget organisms, dust intake itself can contribute to overexposure. Our estimates indicate that, under both an acute and chronic scenario, exceeding the HBGV $($ MOS $=0.1-0.3)$ is pertinent. (Table 2). For Fipronil, at present, sparse evidence exists on intoxication in kittens from the pharmacosurveillance system in place in the European Union. This can be attributed to the following factors: a) Fipronil acute intoxications usually do not lead to severe symptoms, and the reported inappetence, anorexia, vomiting, diarrhea in kittens could be easily confounded with the more frequent perinatal clinical outcomes, i.e., from roundworms present as a

result of prenatal exposure; ${ }^{[32]}$ b) the HBGVs for Fipronil account for a conservative uncertainty factor of 100 (EFSA, 2012); and c) underreporting may occur due to the improvable networking between vet practitioners and the veterinary antipoisoning centers. This last point could highlight again the relevance of risk-oriented stewardship programs from the Health Authorities to promote a One Health approach in the management of persistent pesticides in pet care.

\section{Conclusions}

The reported widespread use of Fipronil in urban and agriculture contexts, its persistence, and environmental release are supported by the evidence provided from our work, which focused on its use as a veterinary drug in Italy. The evidence suggests its inclusion among the so-called pseudopersistent organic pollutants to receive priority monitoring in environmental and food matrices, as well as in pharmacovigilance and pharmacosurveillance schemes supported by records of veterinary prescriptions. 
Authors declare no conflict of interest.

\section{Acknowledgments}

The work was partially supported by the Italian Ministry of Health grant no. RF-2009-1534860 "ENVI-FOOD." The authors wish to thank Dr. Olga Tcheremenskaia for the support in the QSAR analysis of Fipronil and its products and Prof. Marco Pietra for the availability of body weight records of pets.

\section{References}

[1] EFSA European Food Safety Authority. Conclusion on the peer review of the pesticide risk assessment for bees for the active substance Fipronil. EFSA Journal 2013, 11, 3158 - 3208.

[2] Christen, V.; Kunz, P.Y.; Fent, K. Endocrine disruption and chronic effects of plant protection products in bees: Can we better protect our pollinators? Environ Pollut. 2018, 243 (Pt B), 1588-1601.

[3] ANSES (French Agency for Food, Environmental and Occupational Health \& Safety). Scientific and Technical Support Request No 2017-SA-0178 Maisons-Alfort, 10 August 2017 NOTE of the French Agency for Food, Environmental and Occupational Health \& Safety on a request for scientific and technical support (STS) regarding the health risk assessment concerning the presence of Fipronil in eggs intended for consumption. Consulted on October 20, 2018 at https://www.anses.fr/fr/system/files/AUT2017SA0178.pdf

[4] BfR (Bundesinstitut für Risikobewertung). Health assessment of individual measurements of Fipronil levels detected in foods of animal origin in Belgium BfR Opinion No. 016/2017 of 30 July 
2017 Consulted on October 20, 2018 at https://www.bfr.bund.de/cm/349/health-assessment-ofindividual-measurements-of-Fipronil-levels-detected-in-foods-of-animal-origin-in-belgium.pdf

[5] Hainzl, D.; Cole, L.M.; Casida, J.E. Mechanisms for selective toxicity of Fipronil insecticide and its sulfone metabolite and desulfinyl photoproduct. Chem. Res. Toxicol. 11, 1529- 1535.

[6] Simon-Delso, N.; Amaral-Rogers, V.; Belzunces, L.P.; Bonmatin J.M.; Chagnon, M.; Downs L. Furlan, C.; Gibbons, D.W.; Giorio, C.; Girolami, V.; Goulson, D.; Kreutzweiser, D.P.; Krupke, C.H.; Liess, M.; Long, E.; McField, M.; Mineau, P.; Mitchell, E.A.D.; Morrissey, C.A.; Noome, D.A.; Pisa, L.; Settele, J.; Stark, J.D.; Tapparo, A.; Van Dyck, H.; Van Praagh, J.; Van der Sluijs, J. P.; Whitehorn P.R.; Wiemers, M. Systemic insecticides (neonicotinoids and Fipronil): trends, uses mode of action and metabolites, Environ Sci Pollut Res. 2015, 22, 5-34.

[7] Soo-Jeong, L.; Prakash, M.; Diebolt-Brown, B.; Lackovic, M.J; Mehler, L.N.; Beckman, J.; Waltz, J.; Prado, J.B.; Mitchell, Y.A.; Higgins, S.H., Schwartz, A.; Calvert, G.M. Acute illnesses associated with exposure to Fipronil—surveillance data from 11 states in the United States, 2001-2007. Clinical Toxicology, 2010, 48, 737-744.

[8] Bonmatin, J.M.; Giorio, C.; Girolami, V.; Goulson, D.; Kreutzweiser, D.P.; Krupke, C.; Liess, M.; Long, E.; Marzaro, M.; Mitchell, E.A.D.; Noome, D.A.; Simon-Delso, N.; Tapparo, A. Environmental fate and exposure; neonicotinoids and Fipronil. Environ Sci Pollut Res. 2015, 22, 3567.

[9] Richards, J.; Reif, R.; Luo, Y.; Gan, J. Distribution of pesticides in dust particles in urban environments. Environ. Pollut. 2016, 214, 290-298. 
[10] Ensminger, M. P.; Budd, R.; Kelley, K. C.; Goh, K. S. Pesticides occurrence and aquatic benchmark exceedances in urban surface waters and sediments in three urban areas of California, USA, 2008- 2011. Environ. Monit. Assess. 2013, 185, 3697-3710.

[11] Sadaria, A.M.; Sutton, R.; Moran, K.D.; Teerlink, J.; Brown, J.V.; Halden, R.U. Passage of fiproles and imidacloprid from urban pest control uses through wastewater treatment plants in northern California, USA. Environ Toxicol Chem. 2017, 36, 1473-1482.

[12] Tennekes, H.A. Fipronil in Surface Water: An Environmental Calamity Remaining Under Radar in the Netherlands. J Ecol Toxicol. 2018, 2, 1.

[13] Assalco (Italian Association of Petfood Companies) RAPPORTO ASSALCO - ZOOMARK 2018 ALIMENTAZIONE E CURA DEGLI ANIMALI DA COMPAGNIA (In Italian) Consulted at: http://www.assalco.it/index.php?action=shownews\&id=1\&nid=7461

[14] Teerlink, J.; Hernandez , J.; Budd, R. Fipronil washoff to municipal wastewater from dogs treated with spot-on products Science of the Total Environment, 2017, 599-600, 960-966.

[15] Bigelow Dyk, M.; Liu, Y.; Chen, Z.; Vega, H.; Krieger, R.I. Fate and distribution of Fipronil on companion animals and in their indoor residences following spot-on flea treatments. J Environ Sci Health B, 2012, 47, 913-924.

[16] Cochran, R.C.; Liu, Y.; Krieger, R.I.; Ross, J. H. Postapplication Fipronil Exposure Following Use on Pets. J Environ Sci Health A, 2015, 78, 1217-1226. 
[17] Mahler, B.J; Van Metre, P.C.; Wilson, J.T.; Musgrove, M.; Zaugg, S.D.; Burkhardt, M.R. Fipronil and its degradates in indoor and outdoor dust. Environ Sci Technol. 2009, 43, 5665-5670.

[18] Starr, J.M.; Li, W.; Graham, S.E.; Bradham, K.D.; Stout II, D.M.; Williams, A.; Sylvae, J. Using paired soil and house dust samples in an in vitro assay to assess the post ingestion bioaccessibility of sorbed Fipronil. Journal of Hazardous Materials, 2016, 312, 141-149.

[19] FAO (Food and Agriculture Organization) Plant Production and Protection Paper, 163, 2001 Pesticide residues in food - 2000. Report of the Joint Meeting of the FAO Panel of Experts on Pesticide Residues in Food and the Environment and the WHO Core Assessment GroupReport of the 2001 JMPR FAO/WHO Meeting of Experts. Consulted at: http://apps.who.int/pesticide-residuesjmpr-database/pesticide?name=FIPRONIL

[20] McMahen, R.L.; Strynar, M.J.; Dagnino, S.; Herr, D.W.; Moser, V.C.; Garantziotis, S.; Andersen, E.M.; Freeborn, D.L.; McMillan, L.; Lindstrom, A.B. Identification of Fipronil metabolites by time-of-flight mass spectrometry for application in a human exposure study. Environment International, 2015, 78, 16-23.

[21] Guo, Q.; Zhao, S.; Zhang, J.; Qi, K.; Du, Z.; Shao, B. Determination of Fipronil and its metabolites in chicken egg, muscle and cake by a modified QuEChERS method coupled with LCMS/MS, Food Additives \& Contaminants: Part A, 2018, 35, 1543-1552.

[22] US-EPA (U.S. Environmental Protection Agency) Exposure Factors Handbook: 2011. Edition National Center for Environmental Assessment, Washington, DC. 2011, EPA/600/R-09/052F. Consulted at http://www.epa.gov/ncea/ef . 
[23] EU-ECHA (European Union Chemical Agency) 2009 Guidance on information requirements and chemical safety assessment Chapter R.15: Consumer exposure estimation https://echa.europa.eu/documents/10162/23047722/r15_update_version_2_rev20_en.pdf/ea08eca8$\underline{\mathrm{c} 0 \mathrm{f} 9-41 \mathrm{a} 0-9 \mathrm{a} 3 \mathrm{f}-\mathrm{fdab} 925151 \mathrm{~b} 1}$

[24] Chow, K,; Hearn, L.K.; Zuber, M.; Beatty, J.A.; Mueller, J.F; Barrs, V.R. Evaluation of polybrominated diphenyl ethers (PBDEs) in matched cat sera and house dust samples: investigation of a potential link between PBDEs and spontaneous feline hyperthyroidism. Environ Res. 2015, 136, 173-9.

[25] Salis, S.; Testa, C.; Roncada, P.; Armorini, S.; Rubattu, N.; Ferrari, A.; Miniero, R.; Brambilla, G. Occurrence of imidacloprid, carbendazim, and other biocides in Italian house dust: Potential relevance for intakes in children and pets. J Environ Sci Health B. 2017, 52, 699-709.

[26] EFSA (European Food Safety Authority) Reasoned opinion on the review of the existing maximum residue levels (MRLs) for Fipronil according to Article 12 of Regulation (EC) No 396/2005. EFSA Journal, 2012, 10, 2688 -731.

[27] ISPRA (Istituto Superiore per la Ricerca Ambientale) Monitoraggio nazionale dei pesticidi nelle acque: indicazioni per la scelta delle sostanze . Manuali e Linee Guida (In Italian) 152/2017 ISBN 978-88-448-0825-9. Available at: http://www.isprambiente.gov.it/files2017/pubblicazioni/manuali$\underline{\text { linee-guida/MLG_152_17.pdf }}$ 
[28] Eiden, A. L.; Kaufman, P.E.; Oi, F.M.; Dark, M.J.; Bloomquist, J.R.; and Miller, R.J. Determination of metabolic resistance mechanisms in pyrethroid-resistant and Fipronil-tolerant brown dog ticks Medical and Veterinary Entomology, 2017, 31, 243-251.

[29] Settimi, L.; Davanzo, F.; Bacis, G.; Cossa, L.; Giarletta, A.M.; Moretti, S. National informative system for surveillance of hazardous exposures and poisonings: cases identified in 2014. 9th annual report. Rapporti ISTISAN 18/6 2018, 1-8 (in Italian). Available at: http://old.iss.it/binary/publ/cont/18_6_web.pdf

[30] Heidler, J.; Halden, R.U. Fate of organohalogens in US wastewater treatment plants and estimated chemical releases to soils nationwide from biosolids recycling. J Environ Monit. 2009, 11, 2207-15.

[31] Veterinary Medicine Directorate (VMD) Veterinary Pharmacovigilance in the United Kingdom Annual Review 2016, 2018, available at www.gov.uk/government/publications

[32] Peregrine, A.S.; Roundworms in Small Animals. In “ The Merck Veterinary Manual” available on line at: https://www.msdvetmanual.com/digestive-system/gastrointestinal-parasites-of-small$\underline{\text { animals/roundworms-in-small-animals }}$ 
Table 1. Statistical descriptors of the Fipronil (F), Sulfone (FS), and Desulfinyl (FD) occurrence (ng/g) in residential house dust in the absence (above) and in the presence (below) of pets.

\begin{tabular}{rrrr}
\hline PET $~$ & F & FS & FD \\
\hline min & 5 & 5 & 5 \\
P25 & 6 & 5 & 5 \\
P50 & 11 & 5 & 5 \\
mean & 1075 & 230 & 156 \\
P75 & 76 & 24 & 5 \\
P95 & 1400 & 295 & 145 \\
max & 60219 & 13909 & 7941 \\
N & 101 & 101 & 101 \\
LCD* & 21 & 57 & 85 \\
\hline & & & \\
\hline PET + & F & FS & FD \\
\hline min & 5 & 5 & 5 \\
P25 & 43 & 20 & 5 \\
P50 & 367 & 164 & 42 \\
mean & 4987 & 757 & 507 \\
P75 & 3175 & 659 & 292 \\
P95 & 31065 & 3648 & 3067 \\
max & 55904 & 6288 & 5607 \\
N & 60 & 60 & 60 \\
LCD* & 2 & 7 & 20 \\
\hline
\end{tabular}

* Values below the LOQ of $10 \mathrm{ng} / \mathrm{g}$ indicated as Left Censored Data (LCD) and computed as Medium Bound ( $1 / 2$ of the LOQ). 
Table 2. Computed Total Fipronil exposure as ng/kg bw per day in toddlers and kittens via dust in the presence of pets, compared with that from animal petting and contaminated food.

\begin{tabular}{|c|c|c|c|c|c|}
\hline Source & $\begin{array}{c}\text { Dust } \\
\text { (intake) }\end{array}$ & $\begin{array}{l}\text { Petting } \\
\text { (dermal } \\
\text { intake) }\end{array}$ & $\&$ & $\begin{array}{l}\text { Food } \\
\text { (intake) }\end{array}$ & Aggregate MOS \\
\hline
\end{tabular}

\begin{tabular}{lccccc}
\hline Toddlers & US-EPA & EU ECHA & & \\
Acute & 333 & 556 & 560 & 6,600 & \\
(worst case) & $(9.0 F A O)$ & & $(5.4 F A O)$ & $(0.5 F A O) ;$ & $(0.4 F A O)$ \\
& & $(16 E F S A)$ & $(16 E F S A)$ & $(1.4 E F S A)$ & $(1.2 E F S A)$ \\
chronic & 20 & 34 & 75 & 10 & \\
& $(10 E F S A)$ & $(5.9 E F S A)$ & $(2.7 E F S A)$ & $(20 E F S A)$ & $(1.7-1.9 E F S A)$
\end{tabular}

\section{Kittens}

$\begin{array}{lcccc}\begin{array}{l}\text { Acute } \\ \text { (worst case) }\end{array} & 26,076 & \text { na } & \text { na } & (0.1 F A O) \\ \text { chronic } & & & & (0.3 E F S A) \\ & 1,633 & \text { na } & \text { na } & (0.1 E F S A)\end{array}$

na: not applicable; Between brackets and in italics - the Margin of Safety (MOS) - referred to the ArfDs of 3,000 and $9,000 \mathrm{ng} / \mathrm{kg}$ bw per day from FAO, ${ }^{[19]}$ and EFSA, ${ }^{[26]}$ respectively, and the ADI of $200 \mathrm{ng} / \mathrm{kg}$ bw per day from FAO and EFSA. Dust exposure in the presence of pets, petting, and alimentary intakes derived from Cochran et al., ${ }^{[16]}$ and BfR. ${ }^{[4]}$ 


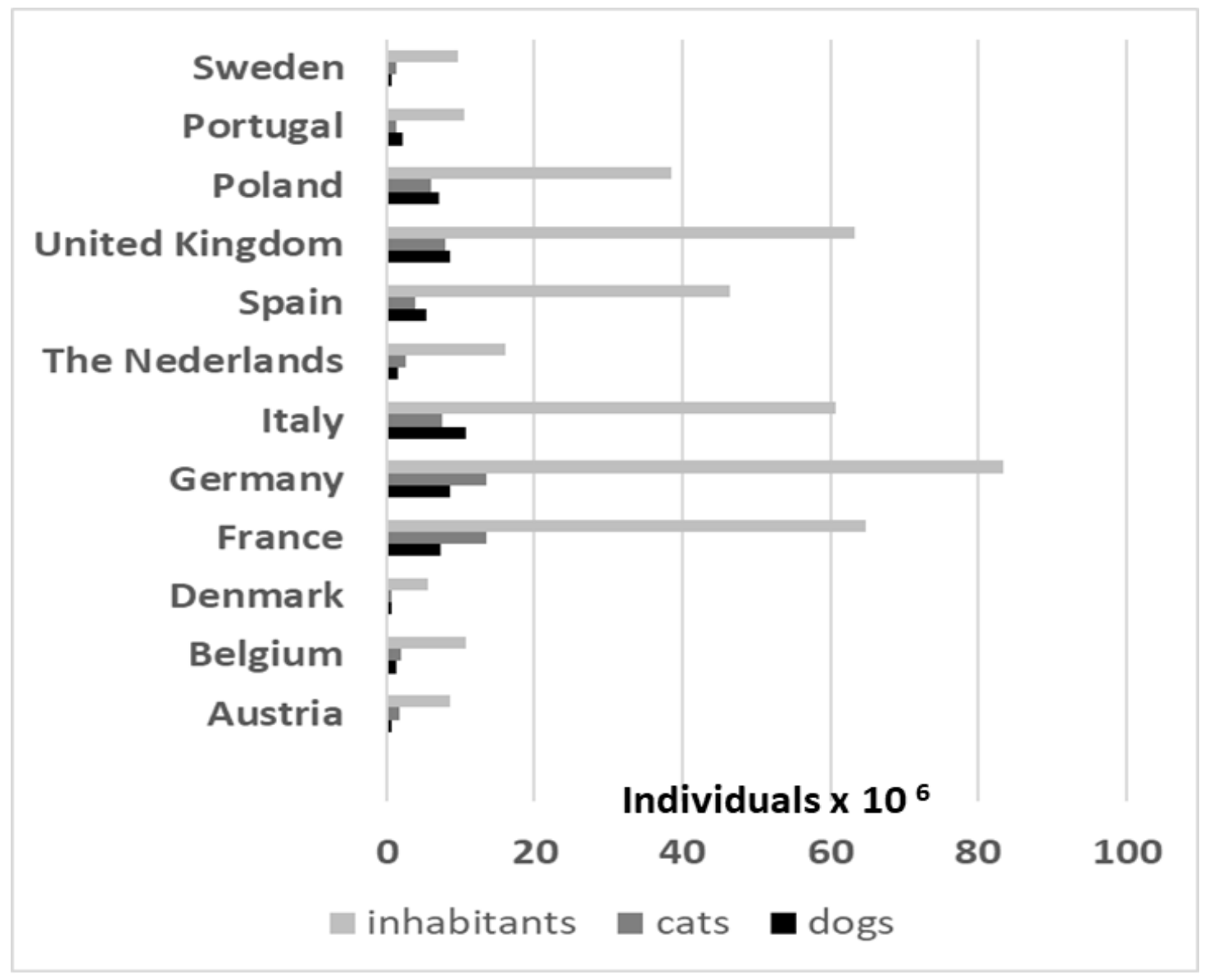

Figure 1. 


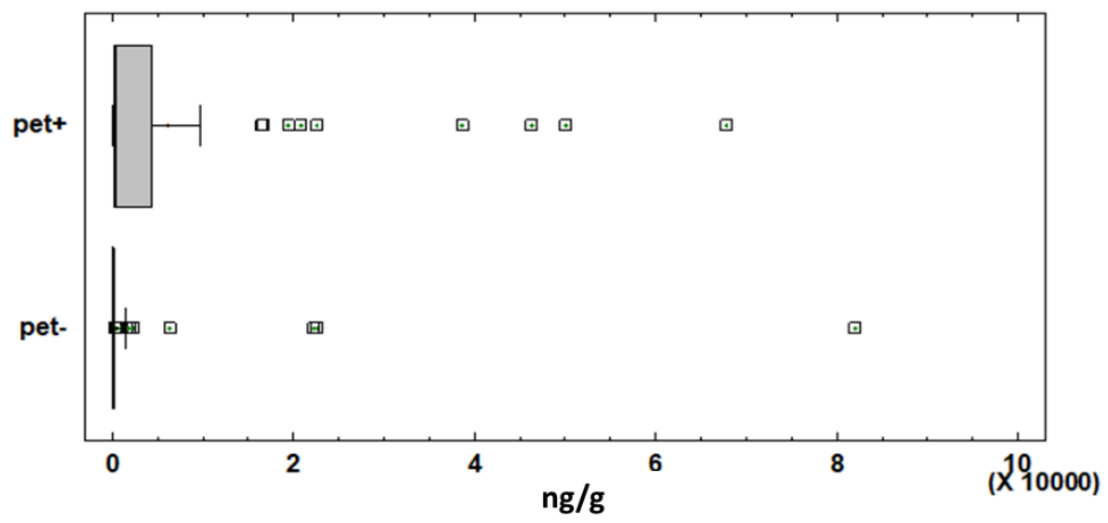

Figure 2.
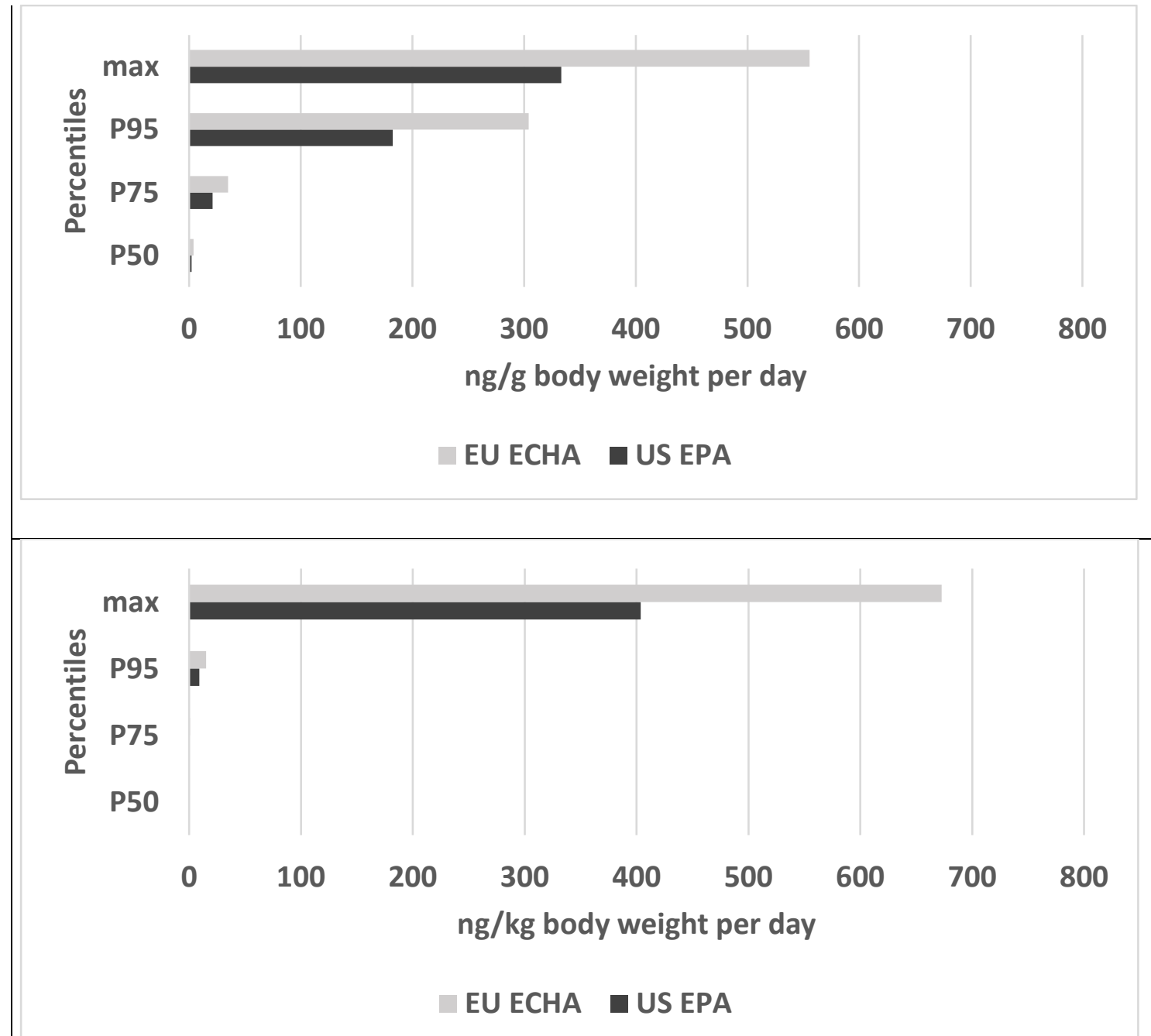

Figure 3 


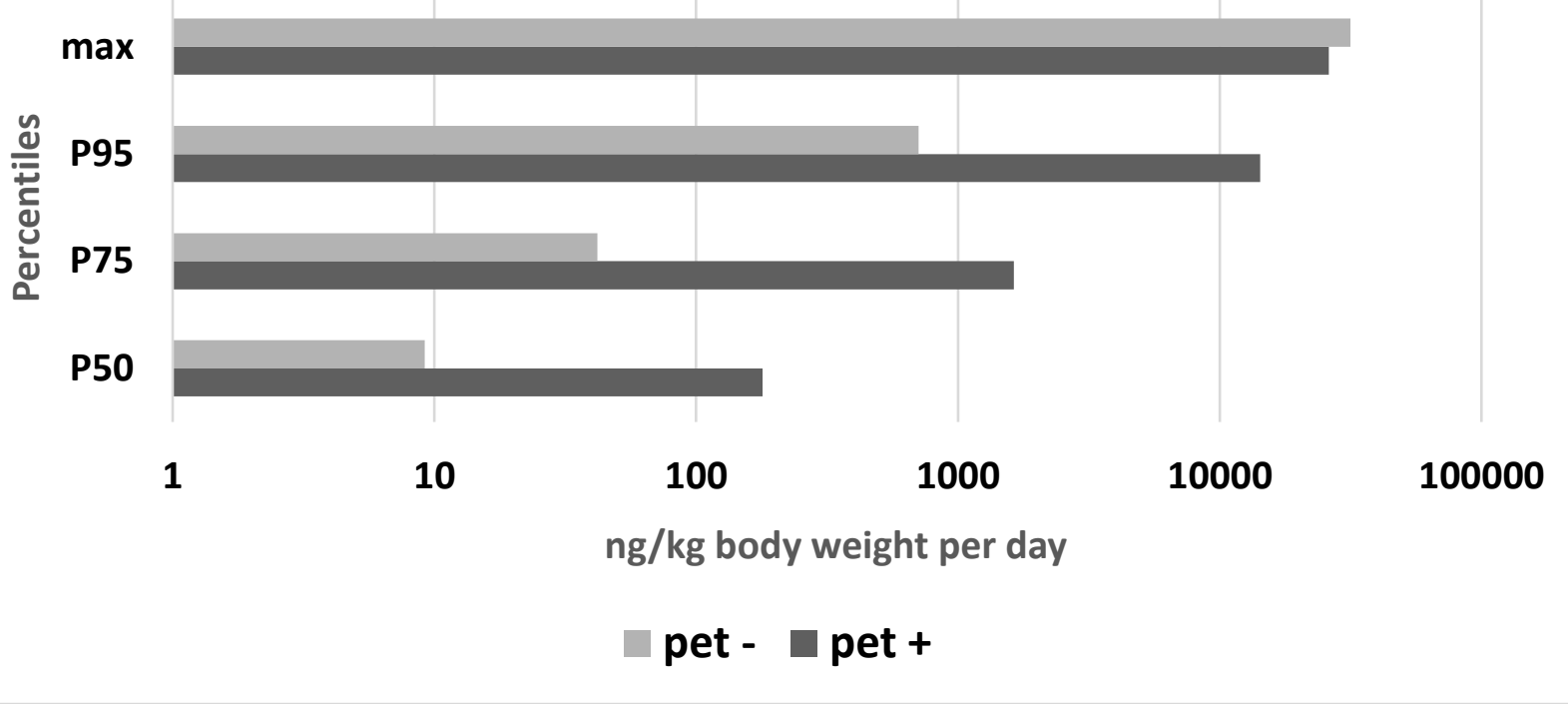

Figure 4.

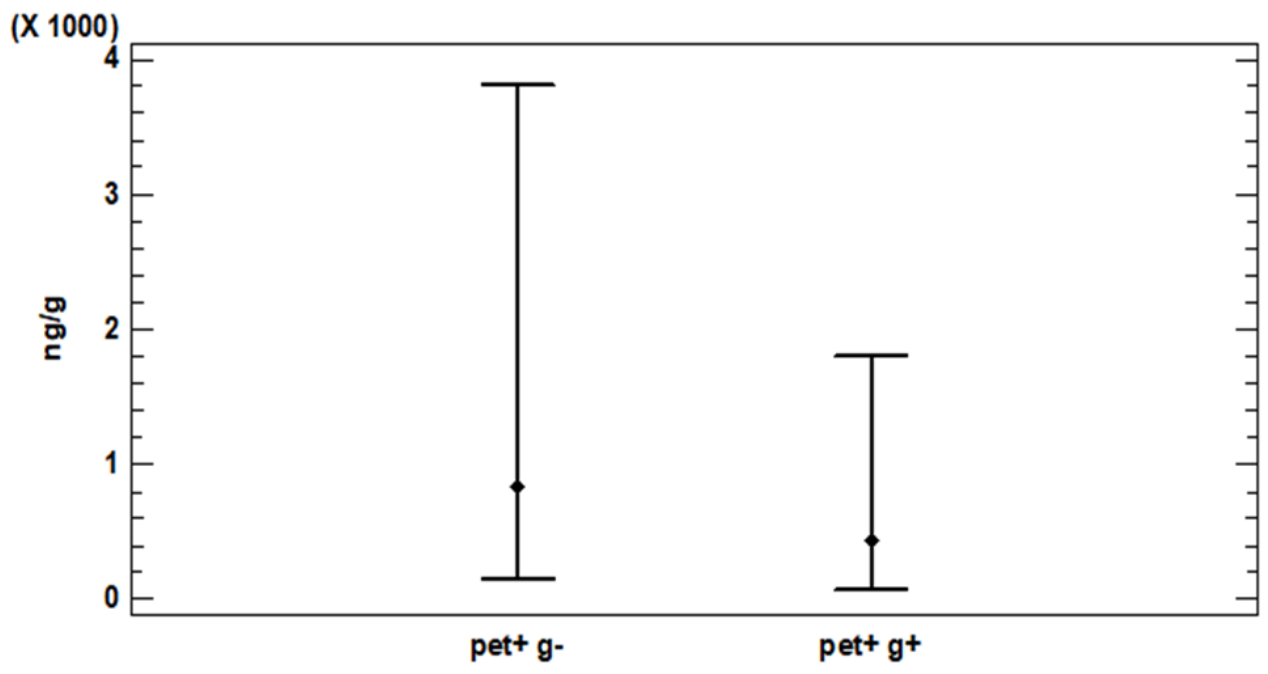

Figure 5 


\section{Figure captions}

Figure 1. Canine and feline population in EU Countries (x $\left.10^{6}\right)$, compared with demographic data, provided by the European Pet Food Industry (FEDIAF, 2017). Dog population in Italy from the national canine registry from the Ministry of Health (2018).

Figure 2. Whisker box-plot output of the total Fipronil house dust contamination $(\mathrm{ng} / \mathrm{g} \times \mathrm{10,000)}$ in the presence (pet + ) and absence (pet -) of cats and dogs.

Figure 3. Intake assessment (ng/g bw per day) of total Fipronil in toddlers according to the US EPA and EU ECHA guidelines from the different occurrence percentiles in house dust in the presence (above) and in absence (below) of pets.

Figure 4. Acute and chronic intake assessment of total Fipronil (ng/g bw per day) in kittens from the different occurrence percentiles in house dust in the presence and in the absence of pets.

Figure 5. Comparison of Fipronil median contamination (ng/g) and of the related $95^{\text {th }}$ percentile confidence intervals in dust in households: a) in presence of pets and of a private garden (pet+ g+) $(\mathrm{N}=38)$; and $\mathrm{b})$ in presence of pets and without a private garden (pet+ $\mathrm{g}-)(\mathrm{N}=22)$. 


\section{SUPPORTING MATERIALS}

Occurrence of Fipronil in residential house dust in presence and absence of pets: a hint for a comprehensive toxicological assessment

CECILIA TESTA ${ }^{1}$, SEVERYN SALIS ${ }^{1}$, NICOLA RUBATTU ${ }^{1}$, PAOLA RONCADA $^{2}$, ROBERTO MINIERO $^{3 a}$, AND GIANFRANCO BRAMBILLA $^{3 b}$

${ }^{1}$ Istituto Zooprofilattico Sperimentale della Sardegna, Department of Food Security, Sassari, Italy

${ }^{2}$ Department of Veterinary Medical Sciences, University of Bologna, via Tolara di Sopra 50, 40064 Ozzano Emilia (BO), Italy

${ }^{3}$ Istituto Superiore di Sanità, ${ }^{a}$ Environment and Health Dept.; ${ }^{b}$ Veterinary Public Health, Food Safety, and Nutrition Dept. Viale Regina Elena, 299 I-00161 Rome, Italy 

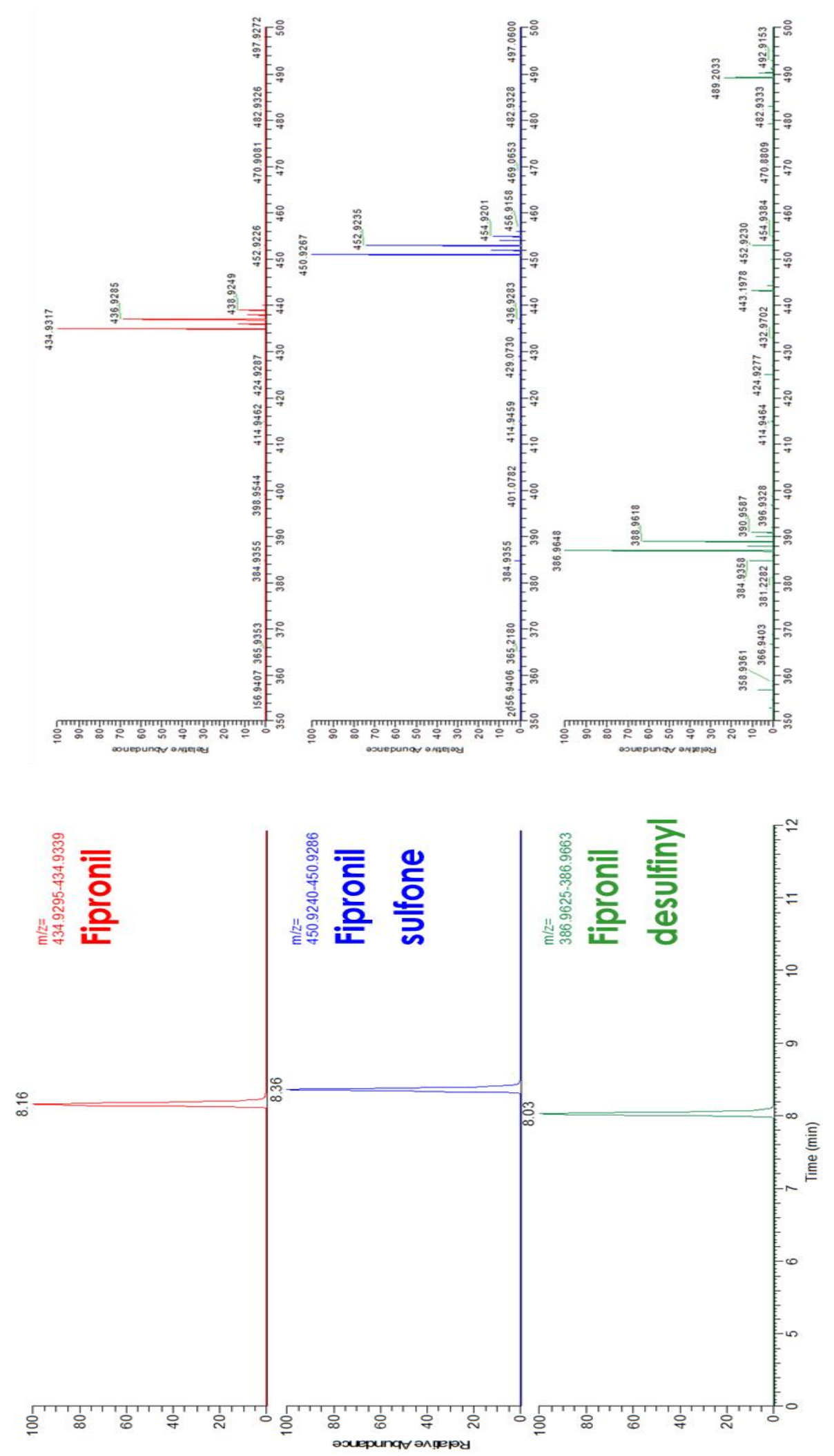

Figure 1S. Chromatograms and High-resolution mass spectra for Fipronil, Sulfone, and Desulfinyl. 
Table 1S. Molecular, retention time, accurate mass, mass errors of Fipronil and its products.

\begin{tabular}{|lccccc|}
\hline Compound & Molecular formula & RT (min) & \multicolumn{2}{c}{ Precursor ion } & Mass error \\
& & & Theoretical Experimental & $(\mathbf{p p m})$ \\
& & & & \\
& & & & \\
& $\mathrm{C}_{12} \mathrm{H}_{4} \mathrm{Cl}_{2} \mathrm{~F}_{6} \mathrm{~N}_{4} \mathrm{OS}$ & 8.16 & 434.9314 & 434.9317 & 0.3 \\
\hline Fipronil & & & & & 0.4 \\
\hline Fipronil-sulfone & $\mathrm{C}_{12} \mathrm{H}_{4} \mathrm{Cl}_{2} \mathrm{~F}_{6} \mathrm{~N}_{4} \mathrm{O}_{2} \mathrm{~S}$ & 8.36 & 450.9263 & 450.9267 & 0.9 \\
\hline
\end{tabular}


Table 2S. Computed use of Fipronil as veterinary drug per year in cats and dogs.

\begin{tabular}{ccccccc}
\hline $\begin{array}{c}\text { Population } \\
\text { dogs (N) }\end{array}$ & $\begin{array}{c}\text { Treated } \\
\%\end{array}$ & $\begin{array}{c}\text { Treated } \\
(\mathbf{N})\end{array}$ & $\begin{array}{c}\text { kg bw } \\
\text { mean }\end{array}$ & $\begin{array}{c}\text { Posology } \\
\text { mg/kg per } \\
\text { month }\end{array}$ & $\begin{array}{c}\text { Treatments } \\
\text { per year } \\
(\mathbf{N})\end{array}$ & $\begin{array}{c}\text { F use } \\
\text { mg per year }\end{array}$ \\
\hline $10,717,479$ & 62 & $6,612,685$ & 17.07 & 6.7 & 8 & $6,273,586,080$ \\
& 82 & $8,756,180$ & & & 8 & $8,307,163,415$ \\
$\begin{array}{c}\text { Population } \\
\text { cats (N) }\end{array}$ & & & & & & \\
$6,967,000$ & 62 & $4,298,639$ & & & 8 & $1,018,399,163$ \\
& 82 & $5,692,039$ & & 6.42 & 8 & $1,348,512,344$ \\
\hline
\end{tabular}

Table 3S. Descriptors of total Fipronil presence in house dust in the presence of pets (pets+), accounting for the availability or not $(\mathrm{g}+/ \mathrm{g}-)$ of a private garden in the household. Test statistic $=$ $0.430356 ; \mathrm{P}$ value $=0.511814$

\begin{tabular}{lllllllll}
\hline & Count & Average & Median & Standard deviation & MAD & Minimum & Maximum & Lower quartile \\
\hline pet+ g- & 22 & 3298.77 & 832.61 & 5756.04 & 772.96 & 15.0 & 22605.9 & 155.91 \\
pet+ g+ & 38 & 7499.18 & 442.48 & 15672.6 & 424.7 & 15.0 & 67798.6 & 54.48 \\
Total & 60 & 6032.37 & 466.7 & 13182.1 & 448.92 & 15.0 & 67798.6 & 75.68
\end{tabular}

\begin{tabular}{llll}
\hline & Upper quartile & Stnd. skewness & Stnd. kurtosis \\
\hline pet+ g- & 3775.49 & 4.92505 & 6.21046 \\
pet+ g+ & 6090.57 & 6.7734 & 8.26557 \\
Total & 4284.5 & 10.0411 & 16.1735 \\
\hline
\end{tabular}

